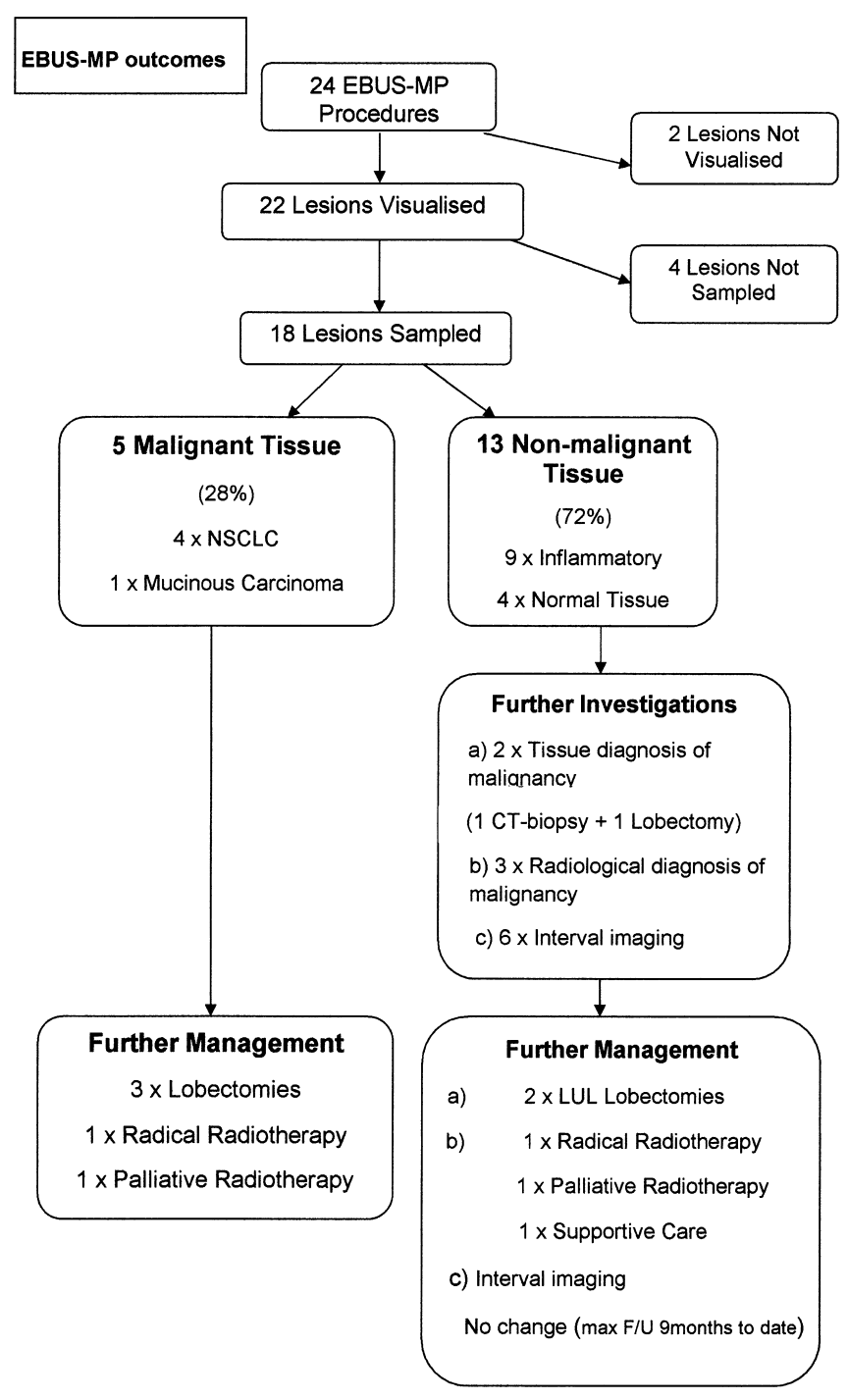

Abstract S40 Figure 1 EBUS-MP outcomes.

a role in investigation of peripheral pulmonary mass lesions. In a well selected cohort of patients it appears to be a safe alternative to CT-guided biopsy.

\section{Immunity and fibrosis in chronic asthma S41 EXPRESSION OF FUNCTIONAL RECEPTOR ACTIVITY MODIFYING PROTEIN (RAMP)-1 BY AIRWAY EPITHELIAL CELLS WITH DYSREGULATION IN ASTHMA}

doi:10.1136/thx.2010.150912.41

A B Kay, K Bonner, H H Kariyawasam, F R Ali, P Clark. National Heart \& Lung Institute, Imperial College, London, UK

Introduction and Objectives Epithelial cell expression of calcitoningene-related peptide (CGRP) is a feature of provoked asthma. Receptor activity modifying protein 1 (RAMP1) and the calcitoninreceptor-like receptor (CRLR) combine to form the CGRP1 receptor. We determined whether functional RAMP1 is expressed by airway epithelial cells and if there are alterations in asthma at baseline and after allergen challenge.

Methods BEAS-2B and A549 cells lines were studied by RT-PCR, confocal microscopy, a quantitative immunofluorescence assay and
ELISA. Bronchial biopsies from normals and asthmatics were examined by immunohistochemistry and in situ hybridisation.

Results Inflammatory cytokines induced CGRP release and CGRP mRNA in BEAS-2B and A549 epithelial cell lines. RAMP1 was highly expressed by resting, unstimulated BEAS-2B and A549 cells. CGRP induced internalisation of RAMP1 and IL- 6 production, both of which were inhibited by the CGRPR antagonist, CGRP8-37. Activation of BEAS-2B and A549 cells by inflammatory cytokines induced CGRP secretion, binding of CGRP to RAMP1 and RAMP1 internalisation which was blocked by CGRP 8-37. RAMP1 immunoreactivity and RAMP1 mRNA expression in bronchial biopsies from asthmatics was significantly lower than in normal subjects ( $p=0.002$ and $p=0.007$, respectively). Inhalational challenge of atopic asthmatics with allergen-derived peptides produced a significant decrease in the numbers of RAMP1-positive epithelial cells in responders $(p=0.027)$ but not non-responders.

Conclusions RAMP1 was expressed both by airway epithelial cells in culture and in bronchial biopsies from normal subjects and internalised after epithelial cell activation through autocrine feedback of CGRP. There is an apparent dysregulation of RAMP1 in asthmatic epithelium suggesting continuous stimulation of pathways involving CGRP.

\section{S42 AIRWAY EPITHELIAL TOLL RECEPTOR EXPRESSION IN ASTHIMA AND ITS RELATIONSHIP TO DISEASE SEVERITY}

doi:10.1136/thx.2010.150912.42

L Cottey, N Jayasekera, H-M Haitchi, B Green, C Grainge, P Howarth. III Research Division, Faculty of Medicine, University of Southamton, Southampton, UK

Introduction Asthma is classically considered a Th2 mediated disease. However, severe and treatment-resistant disease is more heterogeneous and often associated with airway neutrophil recruitment. This may be related to an altered airway bacterial colonisation. Bacteria express pathogen associated molecular patterns (PAMP's) that are recognised as non-self by pattern recognition receptors (PRRs). These PRRs represent an essential component of the innate immunity and an important family of PPRs are the Toll-like receptors (TLR). These are expressed on a range of innate immune cells including epithelial cells. TLR-1, $-2,-4,-5$ and -6 are located on the cell surface membrane and respond to bacterial cell wall components. This study has investigated the expression of mRNA for TLR-2, -4 and -5 in airway epithelial cells in asthmatics and healthy volunteers.

Methods Epithelial brushings were obtained from the large central airways at fibre-optic bronchoscopy from 18 healthy non-asthmatic volunteers ( 8 female and mean age 26 years) and 34 asthmatic volunteers (25 female, mean age 43 years). The asthmatic group comprised 7 non-steroid treated and 27 steroid treated asthmatics. The brushings were placed in Trizol and the RNA subsequently extracted and converted to cDNA, prior to real time TaqMan RT-qPCR analysis for the Toll receptors, TLR2, TLR4 and TLR5, as well as IL-8. Results Gene expression for TLR-2 $(p=0.008)$ and TLR-4 $(p=0.012)$ was significantly increased within the epithelial brushing sample from the asthmatics compared to the healthy control subjects whilst that for TLR-5 did not differ significantly. Interleukin 8 mRNA was also increased within the epithelial brushing sample in the asthmatics $(p=0.007)$ compared to that in the healthy control subjects. These significant differences from the healthy population were also individually present in both the mild and severe asthmatic groups, with no significant difference being evident between mild and severe asthma.

Conclusions These findings reveal up regulation of epithelial gene expression for members of the Toll receptor family relevant to bacterial responses within the airways. Additionally there is enhanced IL-8 gene expression. These features are indicative of on- 